\title{
Modified Integral Sliding Mode Controller Design based Neural Network and Optimization Algorithms for Two Wheeled Self Balancing Robot
}

\author{
Ekhlas karam \\ Al-Mustansirya University/ Computer Engineering Department, Baghdad, 10001, Iraq \\ Email: ek_karam@yahoo.com \\ Noor Mjeed \\ Al-Mustansirya University/ Computer Engineering Department, Baghdad, 10001, Iraq \\ Email: noormjeed @yahoo.com
}

Received: 16 June 2018; Accepted: 13 July 2018; Published: 08 August 2018

\begin{abstract}
Two-wheeled Self-balancing (TWSB) mobile robot is considered to be highly nonlinear and unstable dynamic system. Unstable means that the robot is free to advance forward or backward without any forces applied. It must, therefore, be controlled. The purpose of this work is to design an intelligent nonlinear Modified Integral Sliding Mode Controller (MISMC) based on simple Adaline neural network for balancing a two-wheeled selfbalancing mobile robot, in addition to improve the performance of this robot in tracking the desired trajectory.

The simple Adaline neural network is used to enhance the performance of the conventional Integral Sliding Mode Controller (ISMC) which is an effective and powerful technique because it has a high performance. Also, in this work, a Modified Particle Swarm Optimization (MPSO) and Modified Cuckoo Search (MCS) algorithms have been proposed to find and tune the best MISMC parameters and hence enhance the performance characteristics of the robot system by reducing the processing time as well as improving the response accuracy through minimizing the tracking error of the mobile robot. The Integral Square Error (ISE) method has been used as a performance index for the two algorithms (MPSO, MCS) to measure the performance of the proposed controller. Numerical simulations show the efficiency of the suggested controller by handling the balance and tracking problems of the two-wheeled selfbalancing mobile robot.
\end{abstract}

Index Terms-Modified Integral sliding mode controller, Two-wheeled self-balancing mobile robot, modified PSO, modified CS, optimization algorithms, neural network.

\section{INTRODUCTION}

Two wheeled self-balancing (TWSB) robot is a special type of mobile robotic systems, because of its dynamic system properties are higher nonlinear, unstable, and are not configured so tasks do not perform properly. Compared to another mobile robotics, TWSB mobile robot is characterized by many features, its simple structure, flexible, low cost, small size, and so on $[1,2]$ and also it can be used in different fields with independent different trolley in hospitals and shopping malls, in factories and industrial environments (maintenance electricity services meters, production lines, office and airport, read, used in household activities, airport and office, raising alarms, firefighting, healthcare applications, police patrols, and intelligent robot to disable people or blindly guide) $[3,4]$.

It is known that the TWSB robot suffers from balancing and trajectory tracking problems. The word balance means that the robot is in equilibrium and that its position like standing straight is 90 degrees [5]. However, the system itself is not a balance, which means it continues to fall, away from the vertical axis. In another word, robot trajectory tracking problem is considered as one of important issue aims to track a given time-varying trajectory (reference trajectory). Optimal trajectory tracking for a mobile robot provides a track, which has minimal tracking error and shortest driving time and distance [6].

Many control approaches has been suggested in the literature for balancing the TWSB robot and for improving the ability of this robot in tracking the desired paths, these approaches can be classified to linear and 
nonlinear control approaches. The linear controllers are PID and optimal LQR controller [7, 8], optimal model predictive controller (MPC) as in $[9,10], \mathrm{H}_{\infty}$ controller as in [11].

Different nonlinear controllers (optimal/adaptive) approaches have been suggested to control the TWSB robotic system like sliding mode controllers [12-14], adaptive robust backstepping controller [15], backstepping controller is also combined with PID controller as in [16].

The integral sliding Mode (ISMC) is considered as one of the most common controllers that are often used to control the TWSB mobile robot. Here are some of the previous works; Nguyen and Son [17, 18] design ISMC and LQR for TWSB robot trajectory tracking. A novel implementation of an ISMC for regulation and set point control of a TWSB robot [19].

In this paper, a two vigorous (ISMC and modified ISMC) for TWSB mobile robot are designed because the ISMC controller has the best execution in track the desired trajectory and reject the disturbance. An improved PSO and improved cuckoo search (MCS) algorithm has been proposed to tune the parameters of ISMC and modified ISMC, in order to enhance the performance of these controllers.

The organization of this paper is as follows. In section II, the TWSB robot dynamic model is presented, ISMC design is explained in the section III. Section IV shows the modified ISMC by Adaline NN. Selection parameters based on the MPSO, MCS algorithms are explained in section V. Section VI shows the proposed controller's efficiency with simulation results. Finally, conclusions are provided in section VII.

\section{BALANCING RoBot DYNAMIC MODEL}

In order to design a control unit successfully and to guide (steer) the robot to the desired location, it is necessary to describe the mathematical model of TWSB robot. The mathematical model of this robot is described by differential equations, which are derived from Newton's-Euler equations of motion. This model consists of two separate sub-models, namely: the non-linear equations of the inverted pendulum model (chassis of balancing robot) and linear model of the engine (DC motor), these sub-models then integrated to get two nonlinear differential equations (for position and robot angle) [20-23].
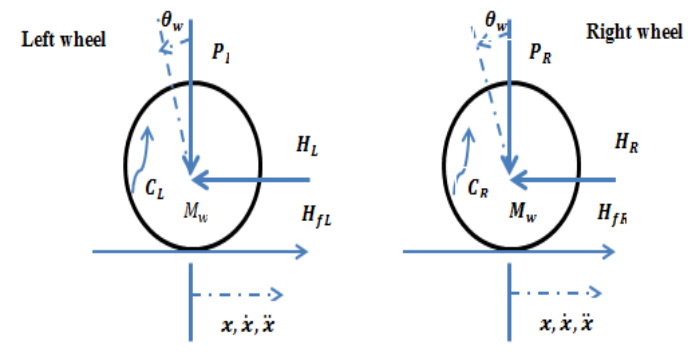

Fig.1. Free body representation of the wheels [23].
Figure(1) represents the free body diagram of the left and right wheels of the TWSB mobile, where $H_{f L}$ and $H_{f R}$ are the force of the friction between ground and wheel and the force of the interaction at the axle interface and rod respectively for the wheel at the right. The dynamic equation of the wheels motion described as [24];

$$
\begin{aligned}
& 2\left(M_{w}+\frac{I_{w}}{r^{2}}\right) \ddot{x} \\
& =-\frac{k_{m} k_{e}}{R r^{2}} \dot{x}+\frac{k_{m}}{R r} V_{a}-\left(H_{R}+H_{L}\right)
\end{aligned}
$$

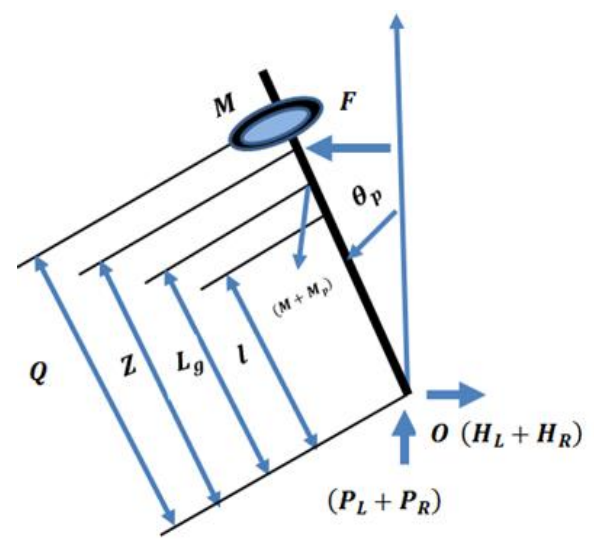

Fig.2. Free body diagram of the chassis [23].

Figure(2) show the diagram free body of the intermediate body (IB) with external disturbance force $(F)$. If the second Newton's law of motion is apply in the horizontal direction results in;

$$
\begin{gathered}
\sum F_{x}=\left(M+M_{p}\right) \ddot{x} \\
\left(H_{L}+H_{R}\right)-\left(M+M_{p}\right) L_{g} \ddot{\theta}_{p} \cos \theta_{p} \\
+\left(M+M_{p}\right) L_{g} \dot{\theta}_{p}^{2} \sin \theta_{p}-F=\left(M+M_{p}\right) \ddot{x}
\end{gathered}
$$

Considering the sum of the applied forces in a direction perpendicular to the rod gives

$$
\begin{gathered}
\sum F_{x}=\left(M+M_{p}\right) \ddot{x} \cos \theta_{p} \\
\left(H_{L}+H_{R}\right) \cos \theta_{p}+\left(P_{L}+P_{R}\right) \sin \theta_{p}-\left(M+M_{p}\right) \\
\left(g \sin \theta_{p}-L_{g} \ddot{\theta}_{p}\right)-F \cos \theta_{p}=\left(M+M_{p}\right) \ddot{x} \cos \theta_{p}
\end{gathered}
$$

If the sum of the applied moments is equating around the global center of mass results in,

$$
\begin{aligned}
& \sum M_{o}=I_{g} \alpha-\left(\left(H_{L}+H_{R}\right) \cos \theta_{p}-\left(P_{L}+P_{R}\right) \sin \theta_{p}\right) L_{g} \\
& -\left(C_{L}+C_{R}\right)-F \cos \theta_{p}=\left(Z-L_{g}\right)=I_{g} \ddot{\theta}_{p}
\end{aligned}
$$


The following two nonlinear differential equations (Eq.(5) and Eq.(6)) results in from manipulating the previous equations, equations Eq.(5) and Eq.(6) describing the dynamic motion of the TWSB mobile robot under uncertainties (impact force disturbance on the rod and applied payload) [24]:

$$
\begin{gathered}
\ddot{x}=\frac{1}{\left(2 M_{W}+\frac{2 I_{W}}{r^{2}}+\left(M_{p}+M\right)\right)}\left(-\frac{2 K_{m} K_{e}}{R r^{2}} \dot{x}+\frac{2 K_{m}}{R r} V_{a}-\right. \\
\left.\left(M_{p}+M\right) L_{g} \ddot{\theta}_{p} \cos \theta_{p}+\left(M_{p}+M\right) L_{g} \dot{\theta}_{p}^{2} \sin \theta_{p}-F\right) \\
\ddot{\theta}_{p}=\frac{1}{\left(I_{g}+\left(M_{p}+M\right) L_{g}^{2}\right)}\left(\frac{2 K_{m} K_{e}}{R r} \dot{x}-\frac{2 K_{m}}{R} V_{a}-\right. \\
\left.\left(M_{p}+M\right) g L_{g} \sin \theta_{p}-\left(M_{p}+M\right) L_{g} \ddot{x} \cos \theta_{p}-F Z \cos \theta_{P}\right)
\end{gathered}
$$

where $x$ is linear position, $\theta$ is the lilt angle for TWSB robot, $F$ force disturbance, $M$ payload, $V_{a}$ is applied voltage. The variables of these equations are defined by Table1 and the physical parameters of TWSB mobile robot are taken from [22].

Table 1. The variables with physical parameters for the used TWSB robot model [22].

\begin{tabular}{|c|c|c|}
\hline Symbol & Definition & Parameter \\
\hline$M_{p}$ & Mass of body & $6 \mathrm{~kg}$ \\
\hline$M_{W}$ & Mass of wheel & $0.3 \mathrm{~kg}$ \\
\hline$K_{e}$ & Back EMF constant & $\begin{array}{c}0.0458 \\
\mathrm{Vs} / \mathrm{rad}\end{array}$ \\
\hline$K_{m}$ & Motor torque constant & $\begin{array}{c}0.0458 \\
\mathrm{Nm} / \mathrm{Amp}\end{array}$ \\
\hline$l$ & Length to the body's center of & $0.2 \mathrm{~m}$ \\
\hline$R$ & Nominal Terminal Resistance & $2.49 \Omega$ \\
\hline$r$ & Radius of wheel & $0.077 \mathrm{~m}$ \\
\hline$g$ & Gravity & $9.81 \mathrm{~m} / \mathrm{s}^{2}$ \\
\hline$I_{w}$ & Inertia of the wheel & $0.0017 \mathrm{~kg} . \mathrm{m}^{2}$ \\
\hline$I_{p}$ & Inertia of the body & $0.29 \mathrm{~kg} . \mathrm{m}^{2}$ \\
\hline$x$ & Position of the chassis & $\mathrm{m}$ \\
\hline$\theta_{p}$ & angle of the chassis & $\mathrm{rad}$ \\
\hline$O$ & Center point & \\
\hline
\end{tabular}

To study the effect of the payload location change, let $Q$ as a variable distance for the payload (M) from the IB origin $\mathrm{O}$, as illustrated by Fig.(2). The distance $Q$ can be expressed as multiplication between the rod half-length 1 and $y$ factor as given by [24]:

$$
Q=y l
$$

$$
\left.I_{g}=\frac{M_{p}(2 l)^{2}}{12}+M_{p}\left(L_{g}-l\right)^{2}+M\left(Q-L_{g}\right)^{2}\right)
$$

where y represent the position of the payload with; $\mathrm{y}=0$ (when $\mathrm{M}$ locate at rod lower end) $\mathrm{y}=1$ (when $M$ locate at rod mid-span) $\mathrm{y}=2$ (when M locate at rod upper end)

The location of the global center of mass of the IB will be affected as:

$$
L_{g}=\frac{\left(M_{p}+Q M\right)}{\left(M_{p}+M\right)}
$$

\section{INTEGRAL SLIDING Mode CONTROLler DESIGN}

Integral Sliding Mode Control (ISMC) is a powerful nonlinear controller designed to control nonlinear systems. Also, the ISMC is insensitive to system parameter variations and the external disturbances [24]. The aim of suggested ISMC in this study is to abolish the reaching phase by forcing the sliding mode throughout the whole robot system response and sliding along the switching surface until the original is reached.

With ISMC approach, the equation of motion order is same as the order of the original system as compare classical SMC which reduce the order of original system by one [25]. As well as with ISMC, the system robustness can be guaranteed because the error and its derivatives reach zero value in final trajectory. The major problem of SMC and ISMC is the chattering phenomenon in the control signal. To reduce this phenomenon, several functions like a reverse tangent function, saturation, and may dead zone be used instead of the (sign function) which is commonly use in traditional SMC.

However, In order to design the ISMC for the two wheeled self-balancing robot as shown in Fig.(3), Eq. (5) \&Eq. (6) should be rewrite as:

$$
\begin{gathered}
\ddot{x}=f_{x}\left(x, \theta_{p}, t\right)+b_{x} u(t)+d(t) \\
\ddot{\theta}_{p}=f_{\theta_{p}}\left(x, \theta_{p}, t\right)+b_{\theta_{p}} u(t)
\end{gathered}
$$

where

$$
\begin{gathered}
f_{x}\left(x, \theta_{p}\right)=\frac{1}{\left(2 M_{W}+\frac{2 I_{W}}{r^{2}}+\left(M_{p}+M\right)\right)}\left(-\frac{2 K_{m} K_{e}}{R r^{2}} \dot{x}-\right. \\
\left.\left(M_{p}+M\right) L_{g} \ddot{\theta}_{p} \cos \theta_{p}+\left(M_{p}+M\right) L_{g} \dot{\theta}_{p}^{2} \sin \theta_{p}-F\right) \\
b_{x}=\frac{1}{\left(2 M_{W}+\frac{2 I_{W}}{r^{2}}+\left(M_{p}+M\right)\right)}\left(\frac{2 k_{m}}{R r} V_{a}\right)
\end{gathered}
$$


and;

$$
\begin{aligned}
& f_{\theta_{p}}\left(x, \theta_{p}\right)=\frac{1}{\left(I_{g}+\left(M_{p}+M\right) L_{g}{ }^{2}\right)}\left(\frac{2 K_{m} K_{e}}{R r} \dot{x}-\right. \\
& \left.\left(M_{p}+M\right) g L_{g} \sin \theta_{p}-\left(M_{p}+M\right) L_{g} \ddot{x} \cos \theta_{p}-F Z \cos \theta_{P}\right) \\
& b_{\theta_{p}}=\frac{1}{\left(I_{g}+\left(M_{p}+M\right) L_{g}{ }^{2}\right)}\left(-\frac{2 K_{m}}{R} V_{a}\right)
\end{aligned}
$$

where scalar $u(t) \in \mathrm{R}$ is the control action, $f_{x}(),. f_{\theta_{p}}($. are the nonlinear function of the system position and angle respectively. $b_{x}, b_{\theta_{p}}$ are the control gain for position and angle respectively, and $d(t)$ is the unknown external disturbance.

As shown in Fig.(3), the controller outputs $u_{x}$ and $u_{\theta_{p}}$ are added to form the final control signal $u(t)$.

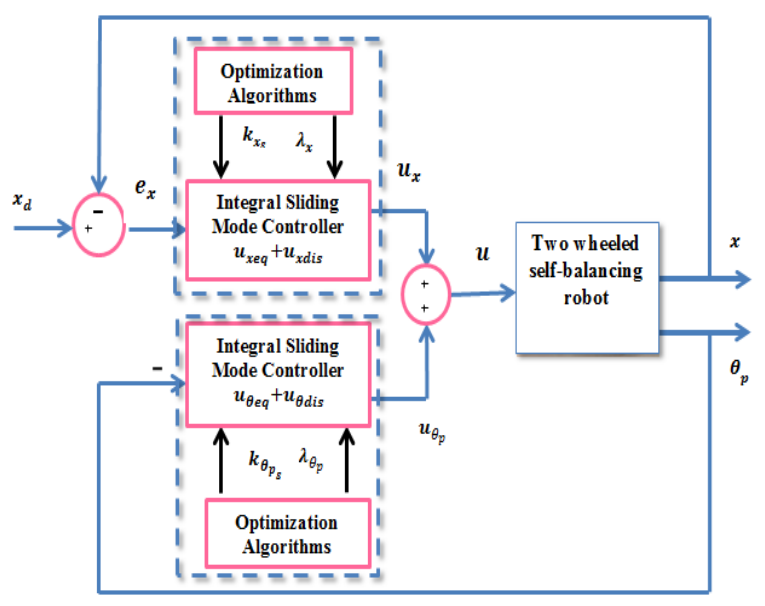

Fig.3. Block diagram of the ISMC with optimization algorithm.

The steps to design of the ISMC $u_{x}$ for balancing robot can be described as follows:

Step one: define the sliding variable $s_{x}$ for position $x$ and angle $\theta_{p}$ as;

$$
s_{x}\left(e_{x}\right)=s_{0_{x}}+z_{x} ; z_{x}(0)=-s_{0_{x}}(0)
$$

where,

$$
s_{0_{x}}=\lambda_{x} e_{x}+\dot{e}_{x}
$$

and

$$
e_{x}=x_{d}-x
$$

The $x_{d}$ is desired trajectory, $\lambda_{x}$ is constant with positive value.

The sliding variable $s_{x}$ consists of two-term; the first term $s_{0_{x}}$ is designed as a linear combination of system states (similar to the conventional sliding mode design), the second term $z_{x}(0)$ introduces the integral term and, is determined based on the initial condition for the sliding variable $s_{0_{x}}(0)$ is zero and this makes the dynamics of the robot in the sliding mode from the first instant.

The sliding variable is derived as in Eq.(19);

$$
\dot{s}_{x}=\frac{\partial s_{0 x}}{\partial e_{x}} \dot{e}_{x}+\dot{z}_{x}
$$

Step two: the ISMC control law $\left(u_{x}\right)$ for robot position is given by;

$$
u_{x}=u_{x_{e q}}+u_{x_{s}}
$$

Here, the first component is the nominal part of controller $u_{x_{e q}}$ which is used to maintain the dynamics of the nominal robot system with the reference properties and second part $u_{x_{s}}$ is discontinuous part of the controller which is used to reject the uncertainty parameters and external disturbance.

By substituting Eq.(10) in Eq.(19)

$$
\dot{s}_{x}=\frac{\partial s_{0 x}}{\partial e_{x}}\left(f_{x}\left(x, \theta_{p}, t\right)+b_{x} u_{x_{e q}}+d(t)\right)+\dot{z}_{x}
$$

If the discontinuous part $u_{x_{s}}$ is define as;

$$
u_{x_{s}}=k_{x} \operatorname{sat}(s)
$$

where $k_{x_{s}}$, is positive value. By included Eq.(22) with Eq.(20), we get;

$$
u_{x}=u_{n_{x}}-k_{x} \operatorname{sat}(s)
$$

Suppose an integrated term is as given by Eq.(24), to satisfy the rejection of system parameter variations and the external disturbances.

$$
\dot{z}_{x}=-\frac{\partial s_{0 x}}{\partial e_{x}}\left(f_{x}\left(x, \theta_{p}, t\right)+b_{x} u_{x_{e q}}\right)
$$

By substituted Eq. (24) in Eq.(21), the following equation is obtained;

$$
\dot{s}_{x}=-\frac{\partial s_{0 x}}{\partial e_{x}}\left(b_{x} u_{x_{s}}+d(t)\right)
$$

The error equation for the position of TWSB robot system can be described as:

$$
\ddot{e}_{x}=-l_{1} e_{x}-l_{2} \dot{e}_{x} ; l_{1,2}>0
$$

The parameters $l_{1}$ and $l_{2}$ are set according to the characteristics required for robot dynamics. 
In ISMC design, the integral term is derived as in Eq.(27);

$$
\dot{z}_{x}=-l_{1} e_{x}-l_{2} \dot{e}_{x}-\lambda_{x} \dot{e}_{x}
$$

Finally, the equation of control law $u_{x}$ for position as below:

$$
\begin{gathered}
u_{x_{e q}}=\frac{1}{b_{x}}\left(\lambda_{x} \dot{e}_{x}-\ddot{x}_{d}-f_{x}\left(x, \theta_{p}\right)+l_{1} e_{x}+l_{2} \dot{e}_{x}\right) \\
u_{x}=u_{x_{e q}}+k_{x_{s}} \operatorname{sat}(s)
\end{gathered}
$$

Repeat steps of Eq.(16) to Eq.(27) for robot angle, so the final control angle law $u_{\theta_{p}}$ will be:

$$
\begin{gathered}
u_{\theta_{p_{e q}}}=\frac{1}{b_{x}}\left(\lambda{\dot{\theta_{\theta}}}_{\theta_{\theta_{p}}}-\ddot{\theta}_{p_{d}}-f_{\theta_{p}}\left(x, \theta_{p}\right)+l_{1} e_{\theta_{p}}+l_{2} \dot{e}_{\theta_{p}}\right) \\
u_{\theta_{p}}=u_{\theta_{p_{e q}}}+k_{\theta_{p_{s}}} \operatorname{sat}(s)
\end{gathered}
$$

The parameters $\left(l_{1, x, \theta_{p}}, l_{2, x, \theta_{p}}, \lambda_{x}, \lambda_{\theta_{p}}, k_{x}, k_{\theta_{p}}\right)$ of the ISMC are tuning based on optimization algorithms as described later in section $\mathrm{V}$.

\section{MOdified INTEGRAL SLIDING MODE CONTROLLER BASED ON ADALINE NEURAL NETWORK}

Adaline is a very simple artificial network containing a layer of input and output with only one neuron, the linear function is used as a transfer function. Fig.(4) illustrates the structure of the Adaline neural network.

The characteristics of this function have rapid learning because of its simplicity and also its ability to solve linear problems $[26,27]$.

$$
W(n+1)=w(n)+\gamma \hat{e}_{n} X_{n}
$$

$\hat{e}_{n}=($ desiredinput $-t \arg e t)$ as shown in Fig.(4), where $\mathrm{y}$ means is net after applied identity (Purlin) activation function, $\mathrm{t}$ is the target, $\gamma$, is the learning rate, $X_{n}$ is input vector of the network and $W(n+1)$ the previous weight vector of the network.

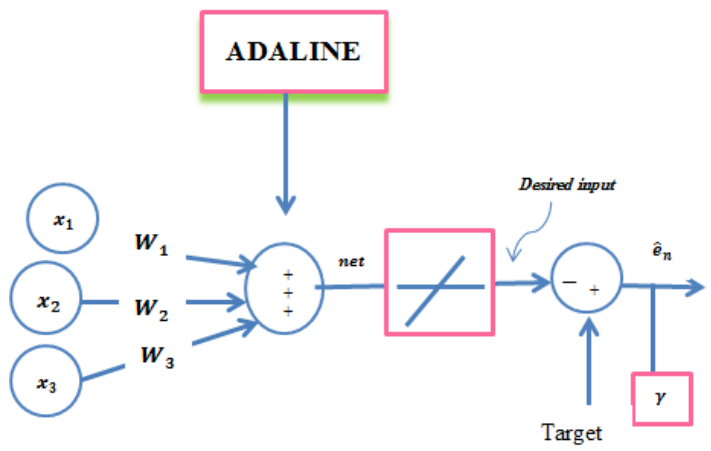

Fig.4. General the structure of Adaline.
In this work, MISMC is designed based on Adaline neural network, where the output of discontinuous part of the ISMC is used to train the Adaline neural network. This method is based on the minimization of Mean Square Error (MSE).

The modification of the discontinuous control part based on an Adaline can be described as below:

The discontinuous part $u_{x_{s}}, u_{\theta_{p_{s}}}$ are the input to the network, and $u_{n x s}, u_{n_{\theta_{p}} s}$ are the output of Adaline NN.

$$
\begin{gathered}
u_{x s}=k_{x s} \operatorname{sat}(s) \\
W(n+1)=w(n)+\gamma u_{x s} \\
u_{n x s}=b+f\left[\sum w(n)+b u_{x s}^{2}\right.
\end{gathered}
$$

where $b$ is constant basis,

$$
\begin{gathered}
u_{x_{c q}}=\frac{1}{b_{x}}\left(\lambda_{x} \dot{e}_{x}-\ddot{x}_{d}-f_{x}\left(x, \theta_{p}\right)+l_{1} e_{x}+l_{2} \dot{e}_{x}\right) \\
u_{x}=u_{x_{e q}}+u_{n x s}
\end{gathered}
$$

Repeat all previous steps for the angle

$$
\begin{gathered}
u_{\theta_{p_{e q}}}=\frac{1}{b_{x}}\left(\lambda_{\theta_{p}} \dot{e}_{\theta_{p}}-\ddot{\theta}_{p_{d}}-f_{\theta_{p}}\left(x, \theta_{p}\right)+l_{1} e_{\theta_{p}}+l_{2} \dot{e}_{\theta_{p}}\right) \\
u_{\theta_{p}}=u_{\theta_{p_{e q}}}+u_{n_{\theta_{p}} s}
\end{gathered}
$$

The ISMC parameters $\left(l_{1, x, \theta_{p}}, l_{2, x, \theta_{p}}, \lambda_{x}, \lambda_{\theta_{p}}, k_{x}, k_{\theta_{p}}\right)$ are tuning based on optimization algorithms as described in next section.

\section{MODIFIED OptIMIZATION AlgORITHM}

The standard stochastic algorithms which use multiple agents (solutions) to move through the search space in the process of solving an optimization problem are known as population-based (or metaheuristic) optimization algorithms. Some of these effective stochastic techniques that mimic the behavior of certain animals or insects (birds, cuckoo) are called Nature-Inspired Algorithms. Two of these techniques (Particle Swarm Optimization and Cuckoo Search Algorithm) are discussed here; these algorithms are experiencing problems and therefore have been improved and modified as we proposed in following subsections

\section{A. Modified Particle Swarm Optimization (MPSO)}

Particle Swarm Optimization (PSO) is a stochastic technique for a population invented in 1995 by $\mathrm{Dr}$. Keendy and Dr. Ebhart. PSO is initially inspired by social behavior of fish schooling or bird flocks. As with other 
population-based algorithms, PSO uses initial random solutions called particles. The best solution in the search space will develop by update generations [28]. If there are $M$ particles (population size), each of which described by three vectors which are; current position $x_{i}^{t}$ vector, velocity $v_{i}^{t}$ vector, and personal best position $p_{i}^{t}$ vector, where these vectors are $N$-dimensional vectors $(1 \leq n \leq N), i$ is the $i^{\text {th }}$ particle $(1 \leq i \leq M)$, and $t$ is the $t^{\text {th }}$ iteration index. These three vectors describe the properties of each individual particle. At the $i^{\text {th }}$ iteration, the best personal best particle according to an objective function $F\left(x_{i}^{t}\right)$ is called the global best particle $p_{g b}$. The update equations of velocity and position vectors are as given by:

$$
\begin{gathered}
v_{i, j}^{t+1}=\operatorname{In}_{w} v_{i n}^{t}+c_{1} r_{1}^{t}\left[p_{i n}^{t}-x_{i n}^{t}\right]+c_{2} r_{2}^{t}\left[p_{g b n}-x_{i n}^{t}\right] \\
x_{i n}^{t+1}=x_{i n}^{t}+v_{i n}^{t}
\end{gathered}
$$

Where $c_{1}$ and $c_{2}$ are the acceleration coefficients, $r_{1}^{t}, r_{2}^{t}$ are a uniform distributed number between [0, 1]. $I n_{w}$ is the inertia weight usually less than one [29]. These random values cause the problem of slow convergence, so this problem has been addressed through a modified method is suggested to adjust the particles velocity and position to overcome the slow convergence problem that emerged in standard PSO algorithm. In this paper, we suggest the following modifications to improve the performance of the PSO algorithm.

1. A Simple Modified Inertia Weight Factor (SMIWF) ' $I n_{w}$ ' is suggested to develop a compromised AIWF that satisfies both exploitation (local search) and exploration (global search). The SMIWF is determined as in Eq. (43) Where $I n_{w}$ must be between (0.4 and 0.9).

$$
\begin{gathered}
s p=\frac{t(\text { currentiteration })}{\max \text { iteration }} \\
I n_{w}(t)=\left(\left(I n_{w_{\max }}-I n_{w_{\min }}\right) * e^{-(s p)^{2}}\right)+I n_{w_{\text {min }}}
\end{gathered}
$$

2. A Simple Modified Acceleration Coefficients (SMACs) $c_{1}, c_{2}$ are suggested to award the efficient particle that has high fitness and punishes the not competent one. These AACs are formulated as in Eq. (44).

Where $c_{1}, c_{2} \leq 2$ must be

$$
c_{1,2}=2-e^{-(s p)^{2}}
$$

3. A Simple Modified Random Variables (SMRNs) $r_{1}, r_{2}$ are suggested to increase the movement impact on the third term (swarm) and decrease the movement influence on the second term This work (individual) of Eq. (40) The equation of SMRVs are formulated as in Eq. (45);

$$
r_{1}, r_{2}=e^{-(s p)^{2}}
$$

where $0 \leq r_{1}, r_{2} \leq 11$ must be

\section{B. Modified Cuckoo Search (MCS) Optimization}

Cuckoo search (CS) algorithm is a search algorithm developed by Xin-she yang and Suash Deb 2009. A new metaheuristic search algorithm, called Cuckoo Search (CS) Optimization, which has gained success in solving complex optimization problems. CSA is a nature-inspired metaheuristic algorithm. CSA essentially based on some cuckoo types brood parasitism and enhanced by levy flights rather than by simple isotropic random walks. In CSA, each cuckoo lays only one egg at a time and put it in a random nest; best nests with higher eggs fitness approved over to next eggs generations. The cuckoo's egg discovered according to probability $\rho_{r} \in[0,1]$, if the host bird discovers the strange egg it will get rid of the cuckoo egg, or abandon the original nest and build another new nest. In CSA, each cuckoo egg represents a candidatethe solution while generating new solutions performed as in Eq. (46);

$$
\begin{aligned}
& n e s t^{t+1}{ }_{i}=n e s t^{t}+s t \oplus \operatorname{levy}(\delta) \\
& i=1,2,3 \ldots, N
\end{aligned}
$$

where $n e s t_{i}^{t}$ is the location of the $t$ generation of the i-th parasitic nest, and st represents the step size vector that is associated to the scales of the optimization problem, $\oplus$ is an entry wise multiplication. Levy flight provides a random walk, while random step performed according to a levy distribution that has an infinite variance and means $[30,31]$.

$$
l e v y \approx u=t^{-\delta}
$$

where $t$ is step size drawn from a Levy distribution. Here, a step size generally forms a random walk with a steplength of low power distribution. Most of the newly generated solutions should be proposed about the optimum solution found so far. Therefore, in this paper, a modified method is suggested to apply an intensive local search to a set of selected solutions to overcome the low diversity of the solution covering near the current global optimal level but not the whole search space, and the slow convergence problem that emerged in Standard CS (SCS) algorithm because use of Levy flight function. Also, The SCS is easy to fall into local boundary optimal value.

Algorithm introduces a modified design which has two search steps 
1. Add a simple improved Inertia Weight Factor (SMIWF) ' $I n_{w}$ ' is suggested to develop a compromised AIWF that satisfies both exploitation (local search) and exploration (global search). The SMIWF is determined as in Eq. (48).

The equation (46) becomes after modification as follow;

$$
n e s t^{t+1}{ }_{i}=\operatorname{In}_{w} n e s t_{i}^{t}+s t \oplus \operatorname{levy}(\delta)
$$

2. Replace the levy function by existing solutions in order to explore the search space near the preserved good solutions (and get the optimal solution in the least time compared with the original algorithm). The equation of replacing is formulated as given by Eq.(49);

$$
n e s t_{i}^{t+1}=\text { In }_{w} \text { nest }_{i}{ }+s t \oplus \frac{\text { rand }[0,1]}{\max \text { iteration }}
$$

\section{SimUlation RESUlT}

With the facility available in the Matlab software version (R2016a), different simulations of the TWSB robot are carried out for linear (step) and nonlinear paths with uncertainties are considered to illustrate the efficiency of the proposed control scheme (MISMC using Adaline NN with MPSO, MCS) as compared with classical (ISMC with MPSO, MCS).

The MCS, MPSO parameters are given in Table 2, and final optimal ISMC, MISMC parameters are given in Table 3, while the MPSO, MCS fitness function ISE is given by:

$$
F=I S E=\int_{0}^{\infty} e^{2} d(t)
$$

Table 2.The parameters that are used in MPSO technique.

\begin{tabular}{|c|c|}
\hline MPSO algorithm & parameters \\
\hline No.of iterations $N_{i}$ & 20 \\
\hline Size of the swarm " no of birds " n & 30 \\
\hline Inertia Weight Factor $I n_{\max }, I n_{\min }$ & $0.9,0.4$ \\
\hline
\end{tabular}

Table 3. The parameters that are used in MCS technique.

\begin{tabular}{|c|c|}
\hline MCS algorithm & parameters \\
\hline No.of iterations $N_{i}$ & 20 \\
\hline No.of nests $n$ & 25 \\
\hline step size $s t$ & 0.01 \\
\hline Discovery rate $\rho_{r}$ & 0.25 \\
\hline Inertia Weight Factor $I n_{\text {max }}, I n_{\text {min }}$ & $0.9,0.4$ \\
\hline
\end{tabular}

Three cases are simulated as follows:

\section{- Simulation result for linear path without uncertainties}

The unit step response of the controlled TWSB robot (position, angle, control signal, and the error signal) are shown in Fig.(5) and Fig.(6). These results show that the performance of robot with MISMC and MCS is more efficient (because ISE is less) than with (MISMC-MPSO, ISMC-MPSO, and ISMC-MCS), where the TWSB robot with MISMC and MCS flow the desired path very fast with very small overshoot and zero steady state error (Fig.6-a), small pitching angle (Fig.6-b), and very smooth control signal (Fig.6-c).

Also, the settling time $t_{s}=2.3$ of the robot response in (MISMC with MCS) is less than the robot response with (MISMC-PSO, ISMC-PSO, ISMC-MCS) controllers as shown in Table 4 and Table 5.

Table 4. Comparison between the proposed controllers with the MPSO algorithm.

\begin{tabular}{|l|l|l|l|l|l|l|}
\hline Controller & $\lambda_{x}$ & $\lambda_{\theta_{p}}$ & $k_{x_{s}}$ & $k_{\theta_{p_{s}}}$ & $t_{s}$ & ISE \\
\hline $\begin{array}{l}\text { ISMC } \\
l_{1_{x}}=3, l_{2_{x}}=1 \\
l_{1_{\theta_{p}}}, l_{\theta_{p}}=1\end{array}$ & 1.2 & 0.8 & 42.7 & 2 & 3.763 & 0.03 \\
\hline $\begin{array}{l}\text { MISMC- } \\
\text { Adaline } \\
l_{l_{x}}=3, l_{2_{x}}=2 \\
l_{1_{\theta_{p}}}, l_{2_{\theta_{p}}}=1\end{array}$ & 1.3 & 1.03 & 3.6 & 1 & 2.37 & 0.007 \\
\hline
\end{tabular}

Table 5. Comparison between the proposed controllers with the MCSalgorithm.

\begin{tabular}{|l|l|l|l|l|l|l|}
\hline Controller & $\lambda_{x}$ & $\lambda_{\theta_{p}}$ & $k_{x_{s}}$ & $k_{\theta_{p_{s}}}$ & $t_{s}$ & ISE \\
\hline $\begin{array}{l}\text { MISMC } \\
l_{1_{x}}=4.3, l_{2_{x}}=1 \\
l_{1_{p}}, l_{2_{p}}=1\end{array}$ & 1 & 1 & 44 & 1 & 3.1 & 0.021 \\
\hline $\begin{array}{l}\text { ISMC-Adaline } \\
l_{1_{x}}=3, l_{2_{x}}=1 \\
l_{l_{\theta_{p}}}, l_{2_{\theta_{p}}}=1\end{array}$ & 1.15 & 1 & 4 & 1 & 2.3 & 0.0064 \\
\hline
\end{tabular}

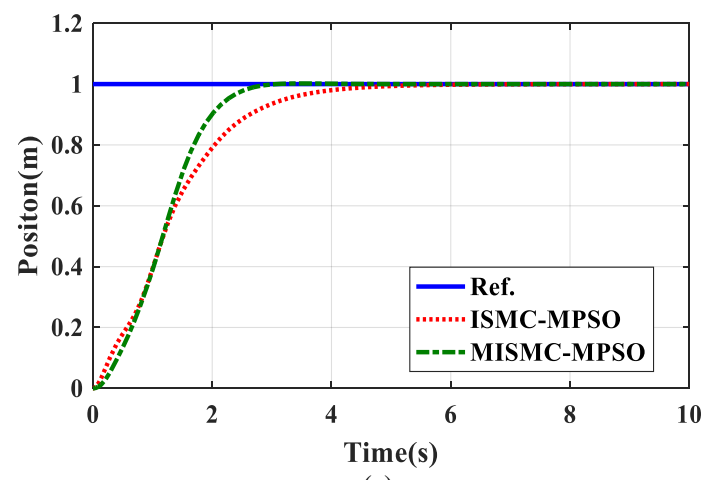

(a) 


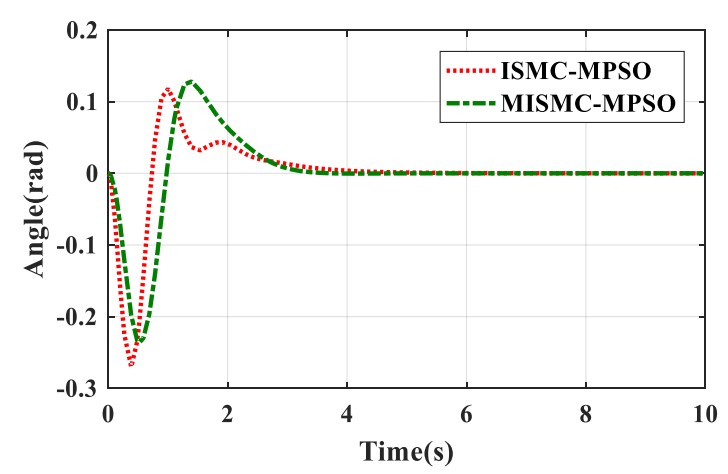

(b)

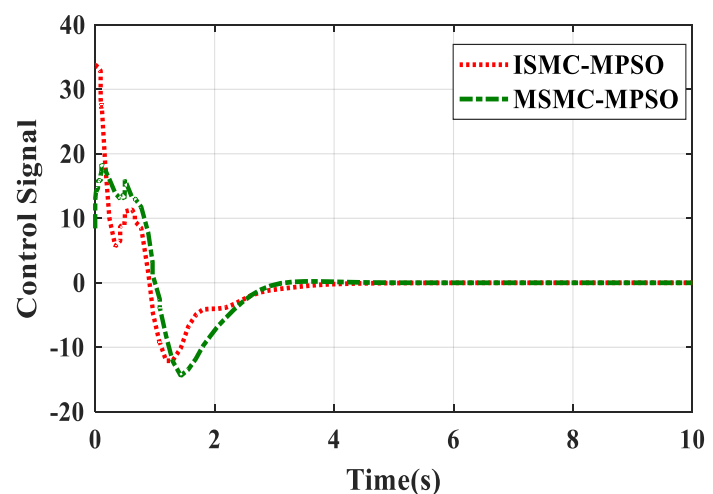

(c)

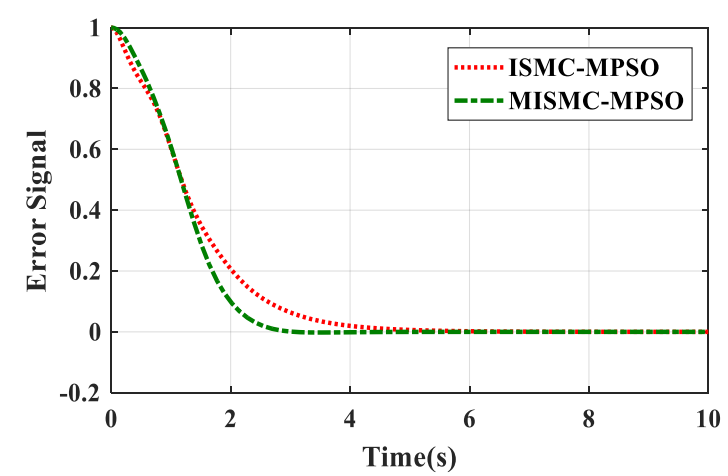

(d)

Fig.5. Simulation result of the TWSB robot controlled by ISMC and MISMC, with MPSO algorithms, (a): Output position, (b): Angle of body, (c): Control signal, and (d): error signal.

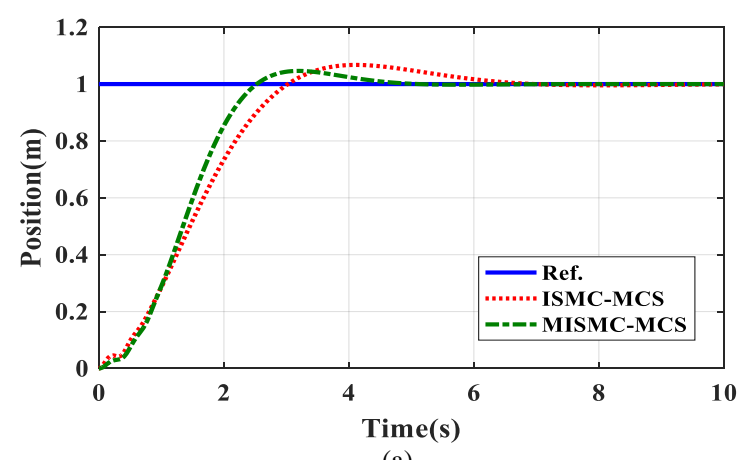

(a)

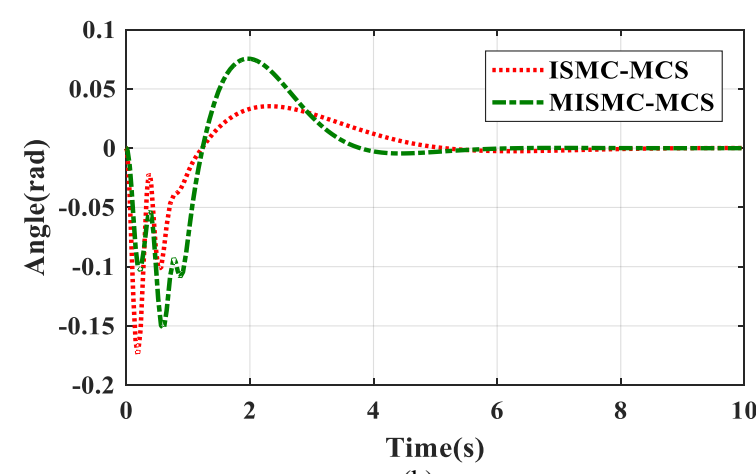

(b)

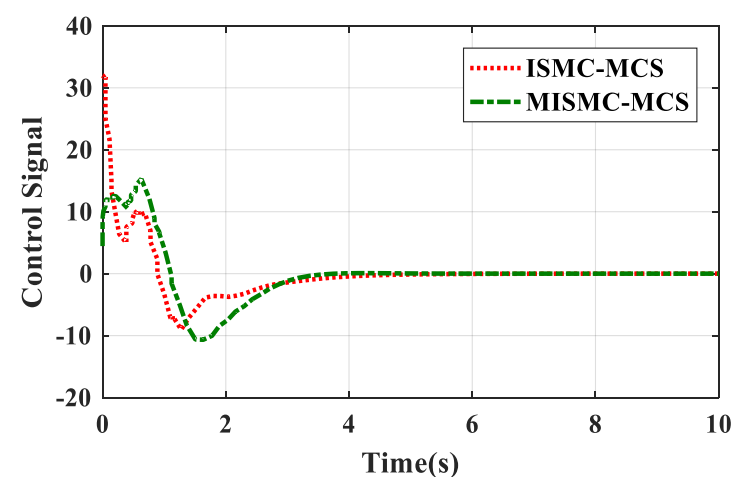

(c)

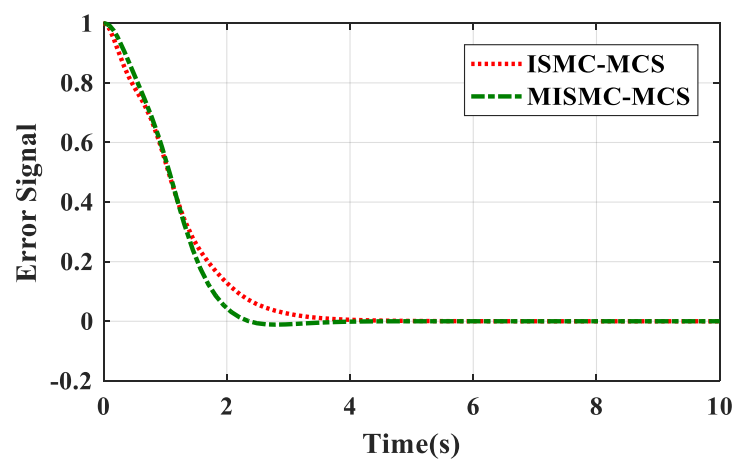

(d)

Fig.6. Simulation result of the TWSB robot controlled by ISMC and MISMC, with MCS algorithms, (a): Output position, (b): Angle of body, (c): Control signal, and (d): error signal.

\section{- Simulation result for nonlinear (sine $\left(x_{d}=0.5 \sin (\pi t / 2)\right)$ ) trajectory (without uncertainties).}

The performance of the TWSBMR with the proposed controller tested (ISMC, MISMC) also with nonlinear input. The best algorithm (MCS only) was applied, the simulation result of sine trajectory tracking of TWSBR model which are shown in Fig. 6 are very good to pose tracking performance achieved by the proposed controller (MISMC) based on (MCS) algorithm compared with ISMC-MCS. 
- Simulation result for nonlinear $\left(x_{d}=0.5 \sin (\pi t / 2)\right)$ path without uncertainties

The performance of the TWSB mobile robot with the proposed (ISMC, MISMC) tested by nonlinear sine input signal and with the best algorithm (MCS only) is illustrated by simulation result shown in Fig.(7), these

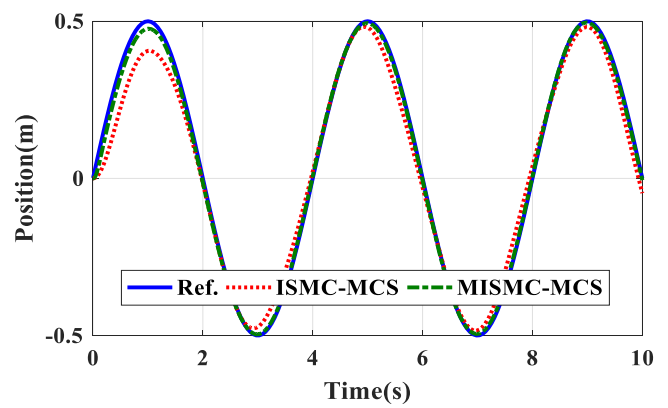

(a)

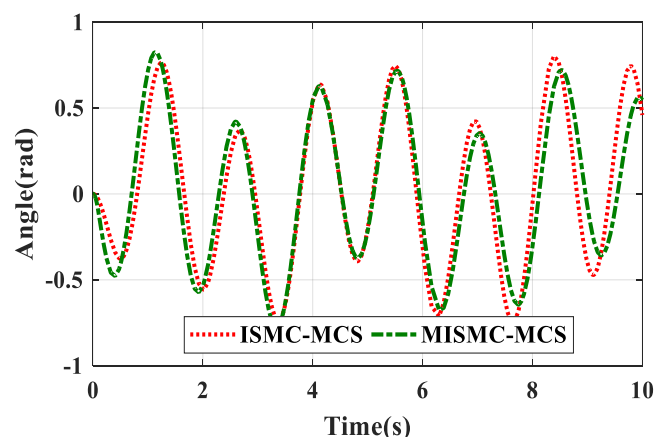

(b)

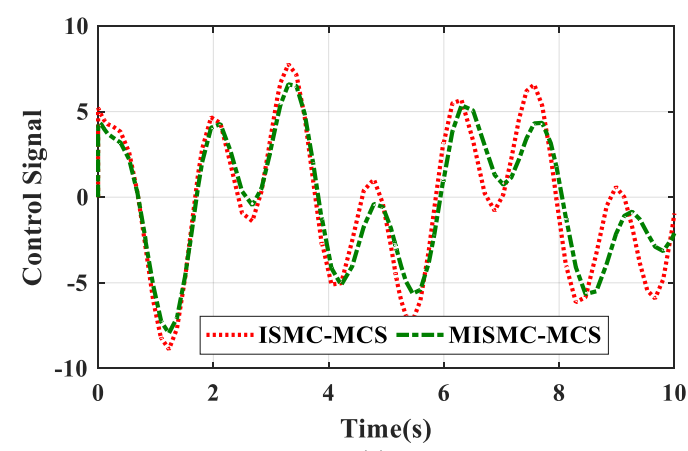

(c)

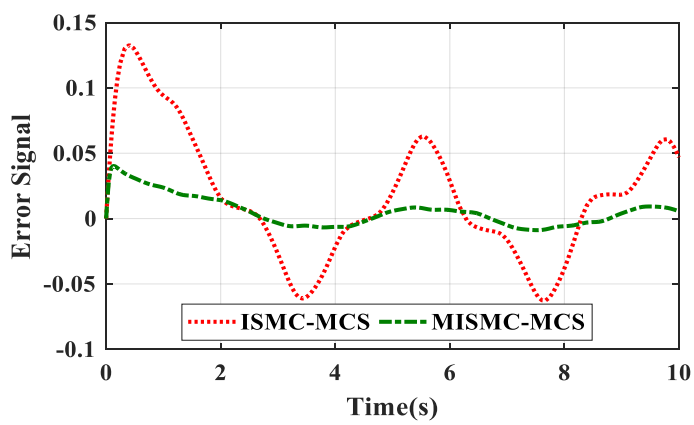

(d)

Fig.7. Simulation result (with sine path) of the TWSB robot controlled by ISMC and MISMC, with MCS algorithms, (a): Output position, (b): Angle of body, (c): Control signal, and (d): error signal. result are very good to pose tracking performance achieved by the proposed controller (MISMC) based on (MCS) algorithm compared to ISMC-MCS.

\section{- Simulation result for linear path with disturbance force $F=30 \mathrm{~N}$ and payload $M=10 \mathrm{~kg}$}

One more time to test the validity of the proposed controller, MISMC-MCS, ISMC-MCS, in coping with uncertainties. The simulations are carried out by considering the disturbance force applied at (upper-end $y$ $=2$ ) positions on the body of the robot

These results show that the TWSB robot is remaining stable even under payload and force disturbance and it flows the desired input signal very fast with no error (Fig.8-a) and small pitching angle (Fig.8-b) and the suitable control signal (Fig.8-c) with the proposed MISMC-MCS based on Adaline NN (the green line) as a compared with ISMC-MCS without Adaline.

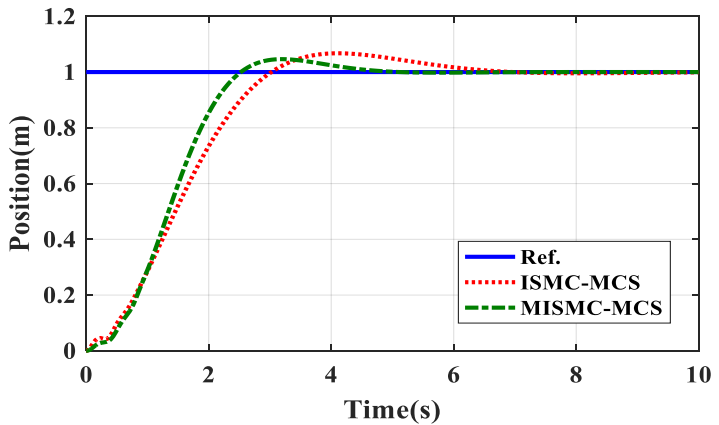

(a)

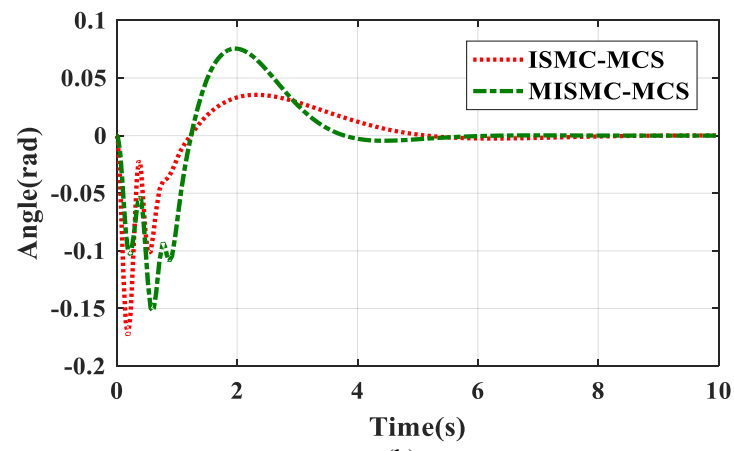

(b)

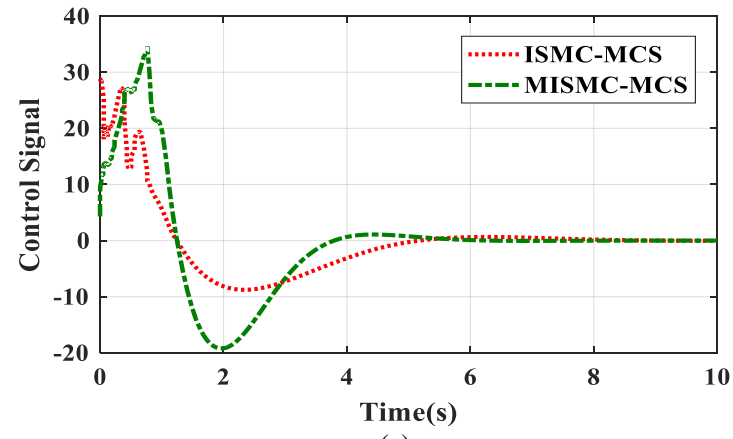

(c) 


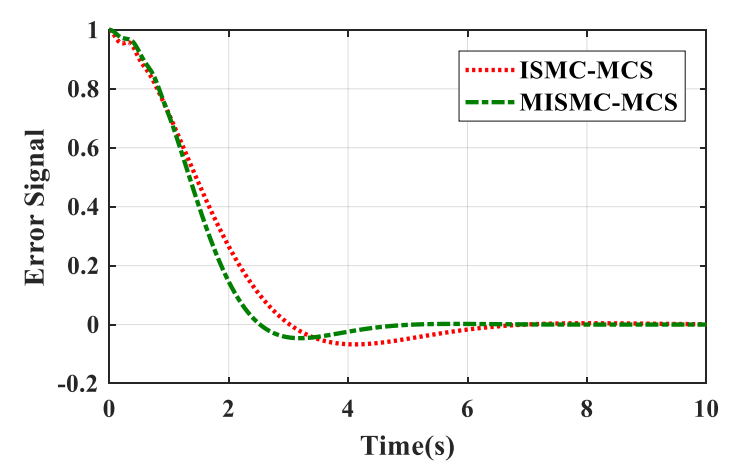

(d)

Fig.8. Simulation result (With $\mathrm{F}=30 \mathrm{~N}, \mathrm{M}=10 \mathrm{~kg}, \mathrm{y}=2$ ) of the TWSB robot controlled by ISMC and MISMC, with MCS algorithms, (a): Output position, (b): Angle of body, (c): Control signal, and (d): error signal.

\section{CONCLUSION}

This work is paying attention to the design non-linear controllers integral SMC and modified integral SMC with Adaline neural network based on proposed (modified PSO, modified CS) algorithms, in order to solve balance problem of the two wheeled self-balancing mobile robots, in addition to improve the performance of TWSB robot in tracking the desired paths. Through the simulation results with the modified cuckoo search (MCS) algorithm has shown better performance and convergence speed rate to an acceptable solution with less number of iterations, and get minimum integral square error (ISE) in ISMC as compared to modified PSO. Also, the simulation results showed the efficiency of the proposed controller (MISMC with MCS) in handling the tracking and balancing problems under uncertainties which gave a high response speed as compared to (ISMC only) with MCS and MPSO.

\section{ACKNOWLEDGMENT}

All thanks and praise be to Allah, the Compassionate, and the Merciful for all His Blessings.

I would like to express my deep sense of gratitude and respect for my supervisor, Asst. Prof. Dr. Ekhlas H. Karam, for the patient, excellent guidance, encouragement, and support she provided throughout my time as his student.

\section{REFERENCES}

[1] S. Chenxi, L.Tao, Y. Kui, "Balance control of twowheeled self-balancing robot based on Linear Quadratic Regulator and Neural Network", International Conference on Intelligent Control and Information Processing (ICICIP ), pp.862-867, June 01, 2013.

[2] L.Sun and J.Gan, "Researching of Two-Wheeled SelfBalancing Robot Base on LQR Combined with PID", International Workshop on Intelligent Systems and Applications (ISA), pp.1-5, May 01, 2010.

[3] H.R.Memarbashi ," Design and Parametric Control of Coaxes Driven Two-Wheeled Balancing Robot", Master
Thesis, Dep. of mechatronics Engineering, New Zealand, the Massey University, June 01, 2011.

[4] C. N. Huang," The Development of Self-Balancing Controller for One-Wheeled Vehicles", Scientific Research Journals of Engineering, Vol. 2,No,4 pp.212$219,2010$.

[5] J. Zhang, G. Li, F. Liu, Y.Liu, "Design of a two-wheeled self-balance personal transportation robot," IEEE Conference on Industrial Electronics and Applications (ICIEA), pp.225-228, June 01, 2016.

[6] Y.Kanayama, Y.Kimura, F. Miyazaki and T. Noguchi ,"A Stable Tracking Control Method for an Autonomous Mobile Robot", Proceedings of IEEE International Conference on Robotics and Automation, No.1, pp.384389, 1990.

[7] H.Juang, K.Lurrr, "Design and Control of a Two-Wheel Self-Balancing Robot using The Adriano Microcontroller Board," IEEE International Conference on Control and Automation, ICCA, pp. 634-639, August 26, 2013.

[8] A.Wei, L.Yangmin, "Simulation and Control of a TwoWheeled Self-balancing Robot", IEEE International Conference on Robotics and Biomimetics (ROBIO), pp. 456-461, December 01, 2013.

[9] Zad, Haris Sheh, Abasin Ulasyar, Adil Zohaib, and Syed Shahzad Hussain. "Optimal Controller Design for SelfBalancing Two-Wheeled Robot System", pp.11-16, 2016.

[10] M. M. Azimi, and H. R. Koofigar,"Model Predictive Control for A two Wheeled Self Balancing Robot ", International Conference on Robotics and Mechatronics (ICRoM 2013), pp. 152-157, February 01, 2013.

[11] N. Dan and J. Wang, "Two wheeled robot self-balancing control research," Indonesian Journal of Electrical Engineering and Computer Science, Vol.2 No.3, pp.617$624,2016$.

[12] F. Dai, F. Li, Y. Bai, W. Guo, C. Zong and X. Gao, "Development of a Coaxial Self-Balancing Robot Based on Sliding Mode Control" IEEE International Conference on Mechatronics and Automation (ICMA),pp. 12411246,August 01, 2012.

[13] D.Y. Gao, P.W. Han, D.S. Zhang, and Y.J. Lu,"Study of Sliding Mode Control in Self-Balancing Two-Wheeled Inverted Car, "APPLIED MECHANICSANDMATERIA", LS,241/244,pp.2000-2003, 2013.

[14] Mohd. Fairus Abdollah, "Proportional integral sliding mode control of a two-wheeled balancing robot", Skudai: Universiti Teknologi Malaysia, 2006.

[15] N. N. Son and H. P. H Anh, "Adaptive Backstepping Selfbalancing Control of a Two-wheel Electric Scooter", International Journal of Advanced Robotic Systems, Vol.11, No.10, pp.1-10, January 30, 2014.

[16] G .M. Nguyen, H. N. Duong, H. P.Nguyen," A PID Backstepping Controller for Two-Wheeled Self-Balancing Robot," International Forum on Strategic Technology (IFOST), pp.76-81,October 01, 2010.

[17] G. Z.QIN ,"Development and Control of An underacted Two- Wheeled Mobile Robot," Ph.D. thesis, University of Singapore ,2012.

[18] B. Shilpa, V. Indu and S. R. Rajasree, "Design of an under actuated self-balancing robot using linear quadratic regulator and integral sliding mode controller," 2017 International Conference on Circuit, Power and Computing Technologies (ICCPCT), Kollam, 2017, pp. 16.

[19] J. X. Xu, Z. Q. Guo and T. H. Lee, "Design and Implementation of Integral Sliding-Mode Control on an Underactuated Two-Wheeled Mobile Robot," in IEEE 
Transactions on Industrial Electronics, Vol. 61, No. 7, pp. 3671-3681, July 2014.

[20] K. M. Goher, M. O. Tokhi, and N. H. Siddique," Dynamic Modeling and Control of a Two Wheeled Robotic Vehicle with a Virtual Payload," Arpn Journal of Engineering and Applied Sciences, Vol.6, No.3, pp.7-41, March 01, 2011.

[21] N. D. Cuong, G. T.Dinh, and T. X. Minh, "Direct MRAS based An Adaptive Control System for a Two-Wheel Mobile Robot,".Journal of Automation and Control Engineering, Vol.3,No. 3, pp.201-207, January 01, 2015.

[22] C.C.Tsai, J. Yu. Shang,and H. Min. Shih., "Trajectory tracking of a self-balancing two-wheeled robot using backstepping sliding-mode control and fuzzy basis function networks", IEEE/RSJ International Conference on Intelligent Robots and Systems (IROS 2010) ,39433948, October 01, 2010.

[23] K. Prakash and K. Thomas, and Koshy Thomas, "Study of Controllers for A Two wheeled Self-Balancing Robot", pp.1-7, 2016.

[24] K. M. K Goher and M. O. Tokhi, "Modeling, Simulation and Balance Control of A Two-Wheeled Robotic Machine with Static Variation in Load Position", Proceedings 22nd European Conference on Modelling and Simulation, 2015.

[25] C. A. Yfoulis, A. Muir, and P. E. Wellstead, "A New Approach for Estimating Controllable and Recoverable Regions for Systems with State and Control Constraints", International Journal of Robust and Nonlinear Control, Vol. 12, No. 7, pp. 561-589, 2002.

[26] S. A. Al-Samarraie, A. S. Badri and M. H. Mishary, "Integral Sliding Mode Controller Design for Electronic Throttle Valve", ALkhwarizmi Engineering Journal, Vol. 11, No. 3, pp.1-12, 2014.

[27] A. Yacine, C. Fatima, B. Aissa, "Trajectory tracking control of a wheeled mobile robot using an ADALINE neural network", International Conference on Electrical Engineering (ICEE), pp.1-5. December 01, 2015.

[28] S. Khalid, and B. Dwivedi, "Application of AI Techniques in Implementing Shunt APF in Aircraft Supply System", January 01, 2014.
[29] Y. del valle et al., "Particle Swarm optimization: Basic Concepts Variants and Applications in Power Systems", IEEE Trans. Evol. Comput., Vol. 12, No. 2, pp. 171-195, Apr,2008.

[30] M. Ali Moqbel Obaid, A. Rashid Husain, and Y. Ahmed Alamr, "Intelligent optimal Controller of Active Suspension System Based on Particle Swarm Optimization", Vol. 6, No.3, pp.79-93, November 2014.

[31] A. A. Al-Khafaji and I. Z. Mat Darus, "Controller Optimization using Cuckoo Search Algorithm of a Flexible Single-Link Manipulator" , IEEE Computer Society Washington, pp.39-44, 2014.

[32] C.Qu, and W. He, "A double Mutation Cuckoo Search Algorithm for Solving Systems of Nonlinear Equations", International Journal of Hybrid Information Technology, Vol.8, No.12, pp.433-448, January 01, 2015.

\section{Authors' Profiles}

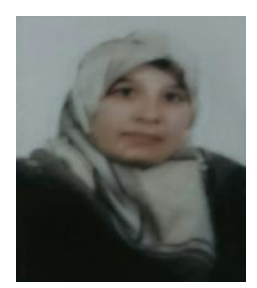

Ekhlas H. Karam, Ph. D, Uni. of Technology, Iraq 2007, M. Sc. Uni. of Technology, Iraq 2001.Academic staff member in Computer Engineering department @Al-Mustansirya University. Interested area: Robotic system, different controller design, optimization methods, image processing, FPGA.

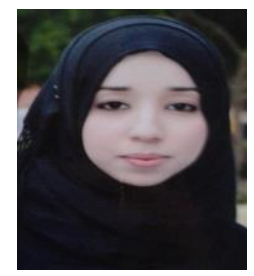

Noor M. Mjeed was born on Oct. 28, 1992. Master of Science student, Computer Engineering dept. @ AL Mustansirya Uni. 2016, B. Sc., Computer Engineering dept. @ AL Mustansirya Uni. 2014. Interested area: Artificial Neural Networks, intelligent algorithms, Optimization Methods, Robotic system, and different controller design.

How to cite this paper: Ekhlas karam, Noor Mjeed, " Modified Integral Sliding Mode Controller Design based Neural Network and Optimization Algorithms for Two Wheeled Self Balancing Robot ", International Journal of Modern Education and Computer Science(IJMECS), Vol.10, No.8, pp. 11-21, 2018.DOI: 10.5815/ijmecs.2018.08.02 